\title{
Solid Pseudopapillary Tumor of the Pancreas: An Enigmatic Tumor
}

\author{
C. Triantopoulou ${ }^{a} \quad$ Z. Touloumis ${ }^{b} \quad$ D. Apessou \\ I. Scotiniotis ${ }^{d}$ D. Rodogiannic J. Papailiou ${ }^{a} \quad$ C. Dervenis ${ }^{b}$ \\ ${ }^{a}$ Computed Tomography Department, ${ }^{b}$ First Surgery Department and \\ 'Pathology Department, 'Konstantopouleion Agia Olga' General Hospital, and \\ ${ }^{\mathrm{d} H y g e i a}$ Hospital, Athens, Greece
}

\section{Key Words}

Pancreas - Neoplasms · Helical computed tomography - Magnetic resonance imaging · Ultrasonography · Biopsy · Fine needle aspiration

\begin{abstract}
Solid pseudopapillary tumor of the pancreas is a rare pancreatic tumor that predominantly occurs in young non-Caucasian women. Although most tumors show benign behavior, malignant degeneration may occur. A case of solid pseudopapillary pancreatic tumor in a Caucasian woman is presented that was investigated by endoscopic ultrasonography (EUS), computed tomography, magnetic resonance imaging and EUS-guided fine needle aspiration. The patient underwent surgery and radiological findings are correlated with histopathology. The preoperative diagnosis of solid pseudopapillary tumor of the pancreas is challenging, frequently leading to imaging by multiple different modalities.
\end{abstract}

\section{Introduction}

Solid pseudopapillary tumor (SPT) of the pancreas is a rare enigmatic tumor of low malignant potential that most frequently occurs in young non-Caucasian women. Although previously suggested, more recent publications have addressed the lack of race predominance. Histogenesis is still uncertain and differentiation could be undetermined. Most tumors are clinically indolent. After complete resection more than $95 \%$ of patients are cured. Because of the degenerative changes that commonly accompany these tumors, they must be considered in the differential diagnosis with other cystic neoplasms and pancreatic pseudocysts. We present a case of SPT in a Caucasian patient investigated by endoscopic ultrasonography (EUS), computed tomography (CT) and magnetic resonance 
imaging (MRI). Radiologic-pathologic correlation is attempted as well as a literature review.

\section{Case Report}

A 32-year-old female Caucasian patient presented to the emergency department of our hospital complaining of postprandial epigastric pain during the last 4 months. She had no history of ulcer or any other pathology of the upper gastrointestinal tract. Physical examination and laboratory tests were unremarkable. Endoscopy of the upper gastrointestinal tract revealed no pathologic findings. The patient underwent EUS followed by CT and MRI of the upper abdomen.

On EUS, a well-circumscribed lesion measuring approximately $3 \mathrm{~cm}$ was confirmed in the pancreatic body. The lesion presented a mixed solid and cystic composition, with frond-like papillary projections into the cystic areas (fig. 1). No surrounding lymphadenopathy was noted, and the pancreatic duct appeared to have a normal course and contour. On contrast-enhanced CT, a well-circumscribed mass with solid and cystic components was revealed in the pancreatic body. Mild enhancement was revealed in the solid parts. The pancreatic duct was slightly dilated in the pancreatic tail (fig. 2). All peripancreatic vessels appeared normal. No peripancreatic collections were revealed. MRI demonstrated a well-defined lesion with heterogeneous (mixed low and high) signal intensity on T1- and T2-weighted images. On T1-weighted image a low intensity area was seen anteriorly. This corresponded to a cystic area that was evident on T2-weighted images presenting high signal intensity. After contrast administration the lesion enhanced partially and gradually but remained hypointense in relation to the normal pancreatic parenchyma ( $\underline{\text { fig. }}$ ).

The patient underwent EUS-guided fine needle aspiration (EUS-FNA), which revealed a homogeneous population of small cells with central 'grooving' of the nucleus, a feature characteristic of SPT. The cell block revealed a papillary architecture, and immunohistochemical stains on the EUS-FNA specimen were strongly positive for vimentin and moderately strongly positive for progesterone receptors. The patient then underwent a distal pancreatectomy with preservation of the spleen. Intraoperatively there was no evidence of local tumor adhesions with adjacent structures, the mesenteric and splenic vessels. The postoperative course was uneventful and she was discharged from hospital after 6 days of recovery. Six months after the operation the patient is free of disease or any kind of symptoms and in excellent general condition.

At gross examination of the surgical specimen a soft, round, well-circumscribed mass with a pseudocapsule was identified. At histologic analysis the tumor was composed of uniform polygonal cells arranged in solid nests with areas of cystic degeneration. The cells were separated into pseudopapillary aggregates ( $\underline{\text { ig. }} 4, \underline{\text { fig. }})$. Areas of old hemorrhage and cholesterol granules were also revealed. No vascular or perineural invasion was identified. Three peripancreatic lymph nodes were negative for metastasis and showed changes of histiocytic lymphadenitis.

Immunohistochemical analysis revealed: vimentin $(+), \operatorname{NSE}(+)$, progesterone receptors $(+)$, CD10 mild (+), pankeratin $(-)$, cytokeratin $7(-)$, chromogranin A (-), synaptophysin $(-)$, Ki67 2\% of the neoplastic cells. Diagnosis was consistent with SPT of the pancreas.

\section{Discussion}

SPT of the pancreas is rare and was first described by Franz in 1959 [1]. Over time many terms have been used to describe the same tumor, such as Frantz's tumor, solid and papillary tumor, papillary cystic tumor, solid-cystic tumor, solid-cystic and papillary epithelial neoplasm, benign or malignant papillary tumor of the pancreas, papillary epithelial neoplasm of pancreas in a child and adenocarcinoma of the pancreas in childhood [2]. WHO in 1996 proposed the name 'solid pseudopapillary tumor' [3]. This term reflects the two most conspicuous histological features: the solid and pseudopapillary areas.

SPTs constitute about $5 \%$ of cystic pancreatic tumors and about $1-2 \%$ of exocrine pancreatic neoplasms. They occur predominantly in girls (not before the age of 9 years) 
and in younger women (mean age 35 years). There is a predilection for Asian and African-American women, but some cases have also been reported in men and children [4]. Although most tumors show benign behavior, malignant degeneration may occur.

These tumors are often older at presentation and have a male predilection [4]. Metastases occur most often in the liver [5]. According to WHO classification, SPTs with clear criteria of malignancy (vascular and nerve sheath invasion or lymph node and liver metastases) are designated as solid pseudopapillary carcinomas. Nevertheless, the tumor is indolent, with long patient survival, even in the presence of extension into adjacent organs or metastases. Patients are usually asymptomatic. In certain cases the tumor may present with a gradually enlarging abdominal mass causing vague abdominal pain or discomfort. Obstructive symptoms may occur if the neoplasm grows large enough or is located in the pancreatic head. Small tumors are usually incidental findings on abdominal imaging studies or during laparotomy performed for other purposes.

Although SPTs may affect any portion of the pancreas, they are slightly more common in the pancreatic tail. This predilection has recently been debated by some authors. In some patients they appear as extrapancreatic tumors with only a localized connection to the gland [6]. In these cases origin from the pancreas may be difficult to show even at surgery [7]. Cases of tumors arising in ectopic pancreatic tissue have also been reported [8]. There are no known associations between SPT and other neoplasms or heritable or acquired medical conditions. Serum amylase levels and cancer markers (CA19-9, carcinoembryonic antigen, $\alpha$-fetoprotein) are usually normal. They are also not associated with paraneoplastic syndromes because of the production of hormones or enzymes by the tumor.

On imaging the combined solid and cystic nature is usually evident. Ultrasonography findings have been described in the literature [9]. Well-encapsulated cystic and solid masses can be seen, but sometimes SPT may present as a solid-looking mass or having internal septa and calcifications. CT usually demonstrates a well-encapsulated lesion with varying solid and cystic components owing to hemorrhagic degeneration. Following contrast material administration, enhancing solid areas are typically noted at the periphery, whereas cystic spaces are usually more centrally located [10]. In our case cystic spaces were located in the anterior aspect of the tumor and solid areas in the posterior. In some instances large tumors show complete cystic degeneration. The cysts often exhibit fluid-debris levels [11]. The presence of a thick peritumoral fibrous capsule results in a radiological similarity to pancreatic pseudocysts.

EUS also typically demonstrates a well-demarcated, encapsulated lesion with mixed solid and cystic components. The diagnostic utility of EUS has been greatly augmented by the ability to perform fine needle aspiration (EUS-FNA) of the lesion. The specimen obtained on EUS-FNA can also be stained immunohistochemically, as was seen in the case presented. However, small SPTs may show no cystic changes at all. In these instances the radiographic appearance could be similar to that of a pancreatic endocrine neoplasm. Areas of high signal intensity on T1-weighted images and low or inhomogeneous signal intensity on T2-weighted images can help identify blood products [12]. Moreover SPTs do not demonstrate the hypervascularity typically seen in islet cell tumors.

In our case hemorrhagic areas were not evident in imaging studies. On the initial contrast-phase the mass was hypointense but showed progressive filling, a finding that has also been described by other authors [4]. Overall, between imaging findings and pathology results, there was agreement in the characterization of cystic areas and in the presence of a peripheral pseudocapsule. 
Although the radiologic features are often informative, significant overlap exists. Fine needle aspiration or biopsy and cytologic or histologic analysis are needed for definitive diagnosis in certain cases. The cytological features of SPT are highly characteristic [13]. On gross examination a solid, cystic, solid-cystic and/or hemorrhagic appearance may be possible, depending on tumor size. Histologically SPT is generally characterized by solid areas with a pseudopapillary pattern and cystic spaces due to degenerative changes. In some cases extensive hemorrhage into the cystic spaces results in the appearance of an organized thrombus. A fibrous pseudocapsule surrounding the tumor may be seen. Some SPTs may be entirely fibrotic whereas others may be entirely cystic.

The differential diagnosis includes acinar tumors, pancreatoblastoma and endocrine tumors. However the immunohistochemical pattern of SPTs is distinctive and differs from that of other primary pancreatic tumors. Cytokeratin expression is rare (in our case it was negative) and markers of acinar differentiation are often negative. Presence of neuroendocrine markers is not significant (chromogranin A is not detected and synaptophysin has a patchy immunoreactivity on 22\%) [14]. In our case there was neither chromogranin nor synaptophysin expression.

Different hypotheses of histogenesis of SPTs have been postulated. Many investigators favor the theory that SPTs originate from multipotent primordial cells, while others suggest an extrapancreatic origin from genital ridges of ovarian-related cells attached to pancreatic tissue during early embryogenesis. Some controversies still exist for both hypotheses. The presence of progesterone receptors like in our case favors the second theory, but SPTs also occur in men without sex hormone abnormalities. A low pool of ki67-reactive tumor cells has also been referred, explaining the good clinical outcome of these tumors, and was also noticed in our case.

Patients with SPTs can be adequately managed by pylorus-preserving pancreatoduodenectomy or spleen-preserving distal pancreatectomy, as in our case [15]. Selected neoplasms of the body can be managed by corpus resection by experienced surgeons dedicated in pancreatic surgery. The efficacy of chemotherapy or radiation therapy for SPT has yet to be shown, although a single case was reported to be resectable after preoperative chemotherapy [16].

Noninflammatory cystic lesions of the pancreas are increasingly recognized due to enhanced imaging modalities. They comprise a wide range of differing underlying pathologies from completely benign through premalignant to frankly malignant. The exact diagnostic and management pathway of these lesions remains challenging and algorithms for the diagnosis and management have been suggested by the International Association of Pancreatology $[17,18]$. SPT should be encountered in the differential diagnosis of a cystic pancreatic lesion with or without a solid component in young patients.

\section{Conclusion}

In view of the relative benign behavior of SPTs, the young age of patients and the complete alleviation of all symptoms after resection, accurate preoperative diagnosis is very important. Radiologists should be familiar with typical and atypical presentation of SPTs and use all available imaging methods in difficult cases. A team of experienced radiologists, gastroenterologists, surgeons and pathologists is essential for the appropriate management of these rare tumors. 


\begin{tabular}{r|l|l|l} 
Case Reports in & $\begin{array}{l}\text { Case Rep Gastroenterol 2008;2:486-493 } \\
\text { D0I: 10.1159/000175566 }\end{array}$ & Published online: December 5, 2008 & $\begin{array}{l}\text { O 2008 S. Karger AG, Basel } \\
\text { ISSN 1662-0631 } \\
\text { www.karger.com/crg }\end{array}$ \\
\hline
\end{tabular}

Fig. 1. EUS image shows a heterogeneous lesion in the pancreatic body with hypo- and hyperechoic features and cystic areas.



Fig. 2. Contrast-enhanced CT reveals a well-circumscribed mass with solid and cystic components and a small calcification. Mild enhancement is seen in the solid parts.






\begin{tabular}{r|l|l|l} 
Case Reports in & $\begin{array}{l}\text { Case Rep Gastroenterol 2008;2:486-493 } \\
\text { D0I: 10.1159/000175566 }\end{array}$ & Published online: December 5, 2008 & $\begin{array}{l}\text { O 2008 S. Karger AG, Basel } \\
\text { ISSN 1662-0631 } \\
\text { www.karger.com/crg }\end{array}$ \\
\hline
\end{tabular}

Fig. 3. T2-weighted MR image. The cystic areas in the anterior aspect of the pancreatic lesion are well delineated from the solid parts posteriorly.



Fig. 4. SPT of the pancreas on the right side of the figure in association with the normal pancreatic parenchyma (HE, $\times 10)$.

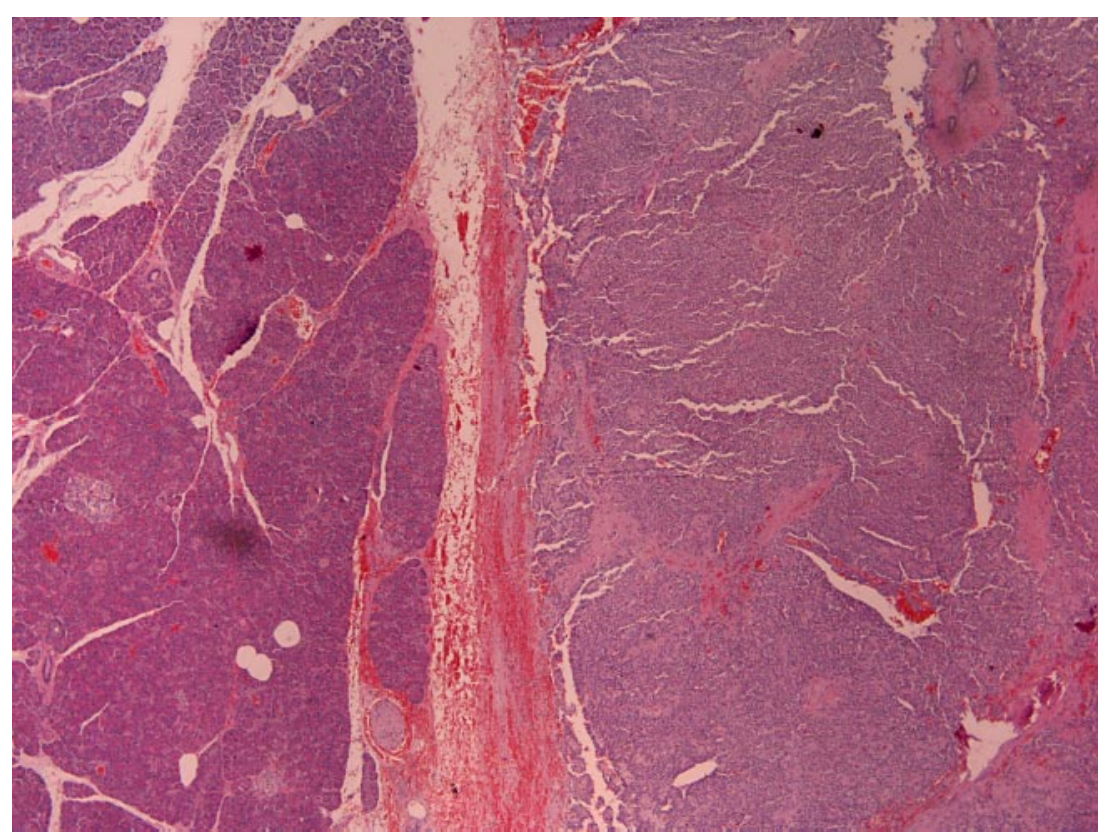




\begin{tabular}{r|l|l|l} 
Case Reports in & $\begin{array}{l}\text { Case Rep Gastroenterol 2008;2:486-493 } \\
\text { D0I: 10.1159/000175566 }\end{array}$ & Published online: December 5, 2008 & $\begin{array}{l}\text { O 2008 S. Karger AG, Basel } \\
\text { ISSN 1662-0631 } \\
\text { www.karger.com/crg }\end{array}$ \\
\hline
\end{tabular}

Fig. 5. The papillary pattern of growth and small cysts of the tumor $(\mathrm{HE}, \times 400)$.

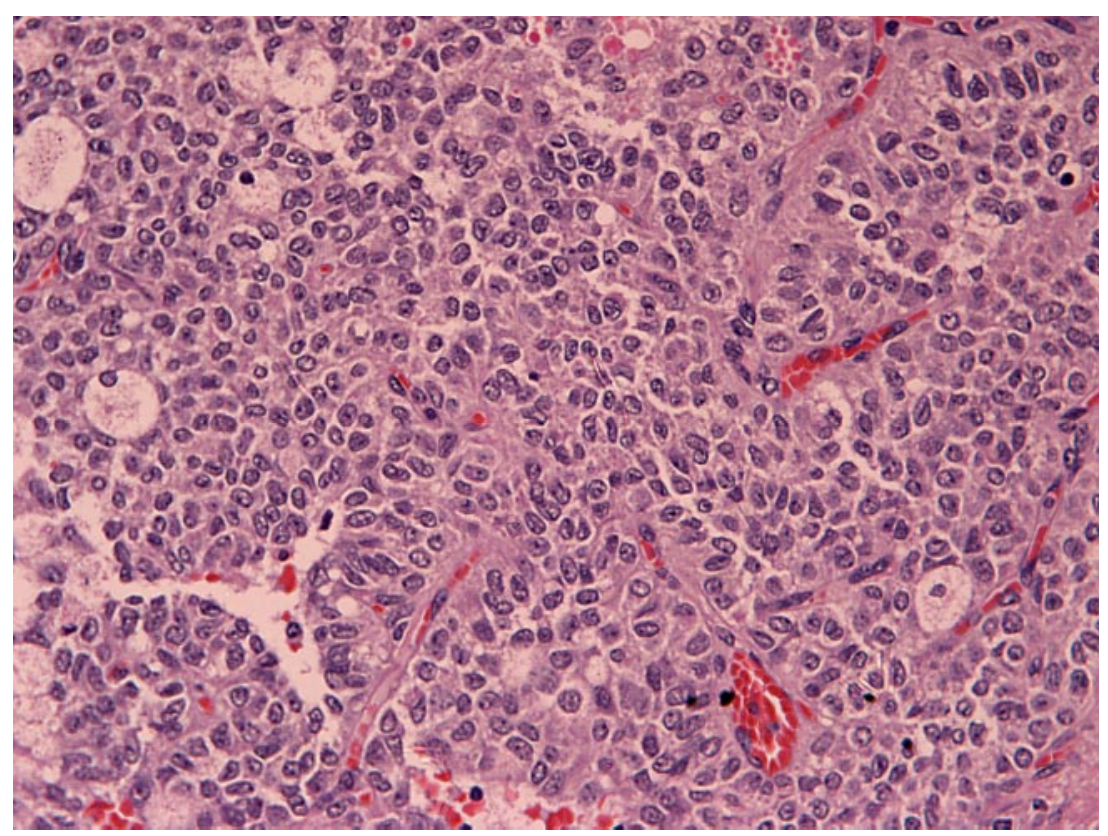




\section{References}

1 Franz VK: Tumors of the pancreas; in: Atlas of Tumor Pathology. Washington, DC, Armed Forces Institute of Pathology, 1959, pp 32-33.

2 Solcia E, Capella C, Kloppel G: Tumors of the pancreas; in: Atlas of Tumor Pathology, 3rd Series. Washington, DC, Armed Forces Institute of Pathology, 1997, pp 120-130.

3 Kloppel G, Luttges J, Klimstra DS, et al: Solid pseudopapillary neoplasm; in Hamilton SR, Altonene LA (eds): World Health Organization Classification of Tumors: Pathology and Genetics of Tumors of the Digestive System. Lyon, IARC Press, 2000, pp 437-480.

4 Coleman KM, Doherty MC, Bigler SA: Solid-pseudopapillary tumor of the pancreas. Radiographics 2003;23:1644-1648.

-5 Sclafani LM, Reuter VE, Coit DG, Brennan MF: The malignant nature of papillary and cystic neoplasm of the pancreas. Cancer 1991;68:153-158.

6 Klimstra DS, Wenig BM, Heffess CS: Solid-pseudopapillary tumor of the pancreas: a typically cystic carcinoma of low malignant potential. Semin Diagn Pathol 2000;17:66-80.

7 Kloppel G, Maurer R, Hofmann E, et al: Solid-cystic tumors within and outside the pancreas in men: report of two patients. Virchows Arch A Pathol Anat Histopathol 1991;418:179-183.

-8 Ishikawa $\mathrm{O}$, Ishiguro $\mathrm{S}$, Ohhigashi $\mathrm{H}$, et al: Solid and papillary neoplasm arising from an ectopic pancreas in the mesocolon. Am J Gastroenterol 1990;85:597-601.

-9 Lee DH, Yi BH, Lim JW, Ko YT: Sonographic findings of solid and papillary epithelial neoplasm of the pancreas. J Ultrasound Med 2001;20:1229-1232.

10 Kehagias D, Smyrniotis V, Gouliamos A, Vlahos L: Cystic pancreatic neoplasms: computed tomography and magnetic resonance imaging findings. Int J Pancreatol 2000;28:223-230.

11 Yamaguchi K, Hirakata R, Kitamura K: Papillary cystic neoplasm of the pancreas: radiological and pathological characteristics in 11 cases. Br J Surg 1990;77:10001003.

12 Cantisani V, Mortele KJ, Levy A, et al: MR imaging features of solid pseudopapillary tumor of the pancreas in adult and pediatric patients. AJR Am J Roentgenol 2003;181:395-401.

13 Kashima K, Hayashida Y, Yokoyama S, et al: Cytologic features of solid and cystic tumors of the pancreas. Acta Cytol 1997;41:443-449.

14 Santini D, Poli F, Lega S: Solid-papillary tumors of the pancreas: histopathology. JOP 2006;7:131-136.

15 De Castro SM, Singhal D, Aronson DC, et al: Management of solid-pseudopapillary neoplasms of the pancreas: a comparison with standard pancreatic neoplasms. World J Surg 2007;31:1130-1135.

16 Strauss JF, Hirsch VJ, Rubey CN, Pollock M: Resection of a solid and papillary epithelial neoplasm of the pancreas following treatment with cis-platinum and 5-fluorouracil: a case report. Med Pediatr Oncol 1993;21:365-367.

17 Garcea G, Ong SL, Rajesh A, Neal CP, Pollard CA, Berry DP, Dennison AR: Cystic lesions of the pancreas. A diagnostic and management dilemma. Pancreatology 2008;8:236-251.

18 Tanaka M, Chari S, Adsay V, Fernandez-del Castillo C, Falconi M, Shimizu M, Yamaguchi K, Yamao K, Matsuno S; International Association of Pancreatology: International consensus guidelines for management of intraductal papillary mucinous neoplasms and mucinous cystic neoplasms of the pancreas. Pancreatology 2006;6:17-32. 